\section{Developing a Mobile Application as an Extension Education Tool: A Case Study Using IPMPro}

\author{
Amy Fulcher ${ }^{1,16}$, Juang-Horng (JC) Chong ${ }^{2}$, Sarah A. White ${ }^{3}$, \\ Joseph C. Neal ${ }^{4}$, Jean L. Williams-Woodward ${ }^{5}$, Craig R. Adkins ${ }^{6}$, \\ S. Kristine Braman ${ }^{7}$, Matthew R. Chappell ${ }^{8}$, Jeffrey F. Derr ${ }^{9}$, \\ Winston C. Dunwell ${ }^{10}$, Steven D. Frank ${ }^{11}$, Stanton A. Gill ${ }^{12}$, \\ Frank A. Hale ${ }^{13}$, William E. Klingeman ${ }^{1}$, Anthony V. LeBude ${ }^{14}$, \\ Karen Rane ${ }^{15}$, and Alan S. Windham ${ }^{13}$
}

AdDitionAl INDEX wORDs. app, smartphone, mobile device, outreach, technology, tablet

Summary. With increased mobile device usage, mobile applications (apps) are emerging as an extension medium, well suited to "place-less" knowledge transfer. Conceptualizing, designing, and developing an app can be a daunting process. This article summarizes the considerations and steps that must be taken to successfully develop an app and is based on the authors' experience developing two horticulture apps, IPMPro and IPMLite. These apps provide information for major pests and plant care tasks and prompt users to take action on time-sensitive tasks with push notifications scheduled specifically for their location. Topics such as selecting between a web app and a native app, choosing the platform(s) for native apps, and designing the user interface are covered. Whether to charge to download the app or have free access, and navigating the intra- and interinstitutional agreements and programming contract are also discussed. Lastly, the nonprogramming costs such as creating, editing, and uploading content, as well as ongoing app management and updates are discussed.

$\mathrm{U}$ se of mobile devices is increasing worldwide; about half of United States cell phone users now have smartphones (Neilsen Co., 2012a) and two-thirds of all new phone purchases are smartphones (Neilsen Co., 2012b). Mobile devices are used for personal, educational, and professional purposes (Bradley, 2010; LaBelle, 2011). Farmers' use of mobile technology is on the rise (Walter et al., 2011) and even outpaces college students' use of technology in some areas (Guenthner and Swan, 2011). Apps may soon become a standard business tool within agriculture as they have in other business sectors (Bradley, 2010).

Apps offer several advantages over traditional forms of delivering Cooperative Extension Service information (Drill, 2012). Agricultural professionals, including extension agents and extension specialists, are often in remote outdoor locations with limited access to traditional sources of information, such as books, extension publications, and computers. Information and resources available through a lightweight, mobile device, particularly via a native app (an app that does not require Internet access but rather runs on the mobile device's operating system and machine firmware) can be an extremely valuable tool. Mobile device apps offer the flexibility of reaching people regardless of location or time of day and offer abundant capacity for data acquisition and storage. Unlike paper publications, apps can be updated any time when there is an Internet connection, allowing extension professionals the ability to quickly disseminate information about emerging issues.

Apps designed as agricultural tools are emerging in the marketplace. Turf Management and Turf Management Lite, the putative first agricultural apps, were launched in 2009 (McCullough et al., 2011). Developers are beginning to incorporate greater sophistication and functionality into new apps. For example, BaiKhao, a recently developed app, estimates the nitrogen level in rice (Oryza sativa) leaves based on device-captured images (Intaravanne and Sumriddetchkajorn,
2012) and IPMPro, launched in 2012 for landscape managers and nursery producers, uses user location to precisely schedule push notifications and has data entry and archiving functions (Fulcher et al., 2012). Opportunities abound for incorporating more sophisticated technology into apps for pest and nutrient management, which will become more important as regulation and management of water and pesticide use are increasingly challenging for nursery growers and other agricultural producers. The objectives of this article are to demonstrate that apps are a promising method for extension professionals to deliver information and to present a summary of the considerations and steps that must be taken to successfully develop an app. For information on the latter stages of app production and launch, please consult the companion article by Fulcher et al. (2013).

\section{Conceptualizing and developing an app for extension audiences}

There are many practical considerations when developing an app. Some initial considerations include finalizing the concept, outlining the functionality and general design, and determining the platform(s). Cost, development and testing time, and technical expertise of the intended clientele are other issues to consider. Administrative time and expense of legal agreements should be evaluated at the onset by the development team. Finally, ongoing hosting costs, content review, and maintenance are recurring issues that will need to be addressed.

EARLY CONSIDERATIONS: MATCHING APP TO CLIENTELE NEED AND TECHNOLOGICAL APTITUDE, PAIRING PLATFORM TO MARKET DEMOGRAPHICS. One of the earliest determinations is deciding whether an app is the best mode of mobile technology that conveys the information and, if so, what type of app is most appropriate (Table 1). There are advantages and disadvantages to both native and webbased apps (Luo, 2010). Apps can be "information presentation" or more interactive, including data entry by the app user. For apps that are information presentation only, it is important to consider the resources that will be expended to make a native app, since the information is likely to be 
already available online on a traditional website. Alternatively, if the information is not already available online, developing a web app (a webpage optimized for viewing on a mobile device) might be the most cost- and time-efficient approach, while still providing relatively similar information delivery and user experience as

The authors thank the University of Tennessee Institute of Agriculture, and UT Research Foundation for financial support received to develop IPMPro and IPMLite, and Diana Cochran and Roy Pargas for their critical review of this manuscript.

This paper was part of the workshop "I Have an App for That: Introduction to Mobile Applications and Development in Horticulture" held on 2 Aug. 2012 at the ASHS Conference, Miami, FL, and sponsored by the Computer Applications in Horticulture (COMP) Working Group.

Mention of a trademark, proprietary product, or vendor does not constitute a guarantee or warranty of the product by the authors or their respective institutions and does not imply their approval to the exclusion of other products or vendors that may also be suitable.

${ }^{1}$ Department of Plant Sciences, University of Tennessee, 2431 Joe Johnson Drive, Ellington Plant Science Building, room 252, Knoxville, TN 37996

${ }^{2}$ School of Agricultural, Forest, and Environmental Sciences, Clemson University, 2200 Pocket Road, Florence, SC 29506

${ }^{3}$ School of Agricultural, Forest, and Environmental Sciences, Clemson University, E-143 Poole Agricultural Center, P.O. Box 340319, Clemson, SC 29634

${ }^{4}$ Department of Horticultural Science, North Carolina State University, 262 Kilgore Hall, Box 7609, Raleigh, NC 27695

${ }^{5}$ Department of Plant Pathology, University of Georgia, 3313 Miller Plant Science Building, Athens, GA 30602

${ }^{6}$ Cooperative Extension Service, North Carolina State University, 120 Hospital Avenue NE, Suite 1, Lenoir, NC 28645

${ }^{7}$ Department of Entomology, University of Georgia, 1109 Experiment Street, Griffin, GA 30223

${ }^{8}$ Department of Horticulture, University of Georgia, 211 Hoke Smith Building, Athens, GA 30602

${ }^{9}$ Department of Plant Pathology, Physiology, and Weed Science, Virginia Polytechnic Institute and State University, 1444 Diamond Springs Rd, Virginia Beach, VA 23455

${ }^{10}$ Department of Horticulture, University of Kentucky, UK Research and Education Center, P.O. Box 469, 1205 Hopkinsville Street, Princeton, KY 42445

${ }^{11}$ Department of Entomology, North Carolina State University, 3318 Gardner Hall, Box 7613, Raleigh, NC 27695

${ }^{12}$ Cooperative Extension Service, University of Maryland, Central Maryland UME, 11975 Homewood Road, Ellicott City, MD 21042

${ }^{13}$ Department of Entomology and Plant Pathology, University of Tennessee, 5201 Marchant Drive, Nashville, TN 37211

${ }^{14}$ Department of Horticultural Science, North Carolina State University, Mountain Horticultural Crops Research and Extension Center, 455 Research Drive, Mills River, NC 28759

${ }^{15}$ Department of Entomology, University of Maryland, Plant Diagnostic Laboratory, 4112 Plant Sciences Building, College Park, MD 20742

${ }^{16}$ Corresponding author. E-mail: afulcher@utk.edu. a native app. One caveat, a web app does not automatically install the app icon on the phone screen as a native app would. Having the icon is an important part of the app experience for many users. The web app icon can be manually installed, but some users will not have the knowledge to install it manually. An Internet connection is essential for web apps but not for native apps. However, a native app that offers in-app purchases or that relies on a web service to access content will require an Internet connection. Both native and web apps can be subscription-based, a one-time fee, or free to download. One advantage of a web app is that the development team retains control over distribution. This can mean larger profits, but more effort is required to distribute the app and to create a payment system. Both Android (Google, Mountain View, $\mathrm{CA}$ ) and iOS (Apple, Cupertino, CA) marketplaces, GooglePlay and iTunes, respectively, have a $30 \%$ transaction fee for all app sales. The remaining $70 \%$ of funds are distributed monthly to the developer.

Understanding the audience and their technological aptitude is critical to a positive user experience. Once the target audience is determined, it is important to match app functionality to the audience. Most apps, whether native or web-based, are largely information presentation, and thereby using and navigating within the app should be straightforward and intuitive. More novel functionality, such as data entry found in IPMPro, requires greater user technological aptitude. Novice app users may be confused by password character requirements, necessity of Wi-Fi or mobile broadband to access some content when a web service is used, and other attributes. Using a focus group composed of people within and outside of the target market is an important step of "ground truthing" the concept, level of functionality, design, and user interface. The focus group session for IPMPro was an integral step during development, helping to ensure that the proposed higher level of functionality was warranted although more expensive, as well as generating additional ideas for increased functionality within future app updates. Just as with county and state extension program planning, stakeholder needs assessment is a fundamental part of app development.
Determining the platform(s) is an early decision in app development that will affect the software programming language and thereby dictate qualifications of the software developer, costs, and suitability to the market. IPMPro was initially slated for development for the Research in Motion (RIM) [now operating under the name BlackBerry (Waterloo, ON, Canada)] market, as well as Google's Android and Apple's iOS (iPhone, iPad, and iPod Touch). However, 1 year later, poor RIM market share (less than $10 \%$ at the time of final programming) made the return on investment too uncertain and app development for the RIM platform was eliminated from the project. Although Android is the worldwide leader in the smartphone market, iOS leads in the United States with $53.3 \%$ of the market share, followed by Android and Windows (Microsoft Corp., Redmond, WA), with $41.9 \%$ and $2.7 \%$, respectively (Moscaritolo, 2013). Individual markets may vary, and state markets may not reflect the national trend. For example, Tennessee nursery growers predominantly use Android and iOS products, with a $45 \%$ market share for each platform, and with a greater share of alternative platforms (10\%) compared with the national average. When developing apps tailored for a specific group, researching the platform of choice could be very beneficial. For example, in the United States there are about three times as many IPMPro users running the Apple operating system compared with the Android system, despite the $11 \%$ market share difference between the two. Tailoring the app platform to these market trends becomes critical when cost concerns limit the number of platforms for which an app can be developed.

USER INTERFACE DESIGN CONSIDERATIONS. Several detailed decisions must be made regarding the user interface (the look and feel of the onscreen menu system "driving" the app experience), and these are target market specific. Flexibility within this user interface is critical, especially during development. The contractual capacity to change the preliminary user interface after testing/simulation of the app with a focus group is very important as mock testing through app simulators will not reveal all issues with the user interface. For example, while developing IPMPro, we learned that 
Table 1. Decision-making chart for determining optimum use of technology that is accessible through mobile devices. Information in the table indicates the optimum but not necessarily the only vehicle for providing stated functionality or feature. In some cases, the most typical use or situation is represented.

\begin{tabular}{|c|c|c|c|}
\hline Desired or key mobile device application (app) feature/function & Website & $\begin{array}{l}\text { Mobile optimized } \\
\text { website (Web app) }\end{array}$ & Native app ${ }^{z}$ \\
\hline One-way communication, app to user & Yes & Yes & Yes \\
\hline Two-way communication, app to and from user & Yes & No & $\mathrm{No}^{y}$ \\
\hline Information available by touchscreen navigation & No & Yes & Yes \\
\hline Unique interactivity, personalization & Yes & No & Yes \\
\hline Wi-Fi or mobile broadband is necessary to access content & Yes & Yes & $\mathrm{No}^{\mathrm{y}}$ \\
\hline Complex calculations & Yes & Yes & $\mathrm{No}^{\mathrm{y}}$ \\
\hline User data entry and archiving & Yes & No & $\mathrm{No}^{\mathrm{y}}$ \\
\hline $\begin{array}{l}\text { Access mobile device's native functionality or processing (access mobile device } \\
\text { camera, global position system, accelerometer, etc.) }\end{array}$ & $\mathrm{N} / \mathrm{A}$ & No & Yes \\
\hline Free access & $\mathrm{Yes} / \mathrm{No}^{\mathrm{w}}$ & Yes/No & Yes/No \\
\hline Developer controls distribution & $\mathrm{N} / \mathrm{A}$ & Yes & No \\
\hline Lower development cost & Yes & Yes & No \\
\hline Lower maintenance cost & Yes & Yes & No \\
\hline
\end{tabular}

${ }^{\mathrm{z}}$ A native app runs on the mobile device's operating system and machine firmware. A native app does not require Internet access, although some optional features of a native app, such as a web service, may require Internet access.

yAnswer yes, or optimized, if a web service is used.

${ }^{\mathrm{x}} \mathrm{N} / \mathrm{A}=$ not applicable.

wSome websites, particularly for periodicals, have fee-based access, but most websites are free.

'If using a web service, content updates can be instantaneous. Downloading the latest version of the app is done manually, not automatically

the bars users touched to trigger display of an individual alert were too small and close together to correctly prompt appearance of the desired alert. This was especially true for males with large fingers, a significant segment of our target market. We also modified the programmer's original pastel color scheme by selecting vibrant, less feminine colors to broaden appeal in our agricultural market base. Demographics of the target market are the single most important factor driving user interface design and will help prioritize design considerations such as color scheme, font, image size, and attribution to contributing institutions. The average nursery crop producer is middle aged, therefore ensuring the app used sufficient font size was very important to our market. Consideration of app appearance in full sun conditions was also important, as the app is intended for use in field settings.

Platform Development costs, PRICING "FOR-FEE" OR "FOR FREE," AND CONTRACTUAL CONSIDERATIONS. Developing an app can be a major investment of time and money. An app that is simplistic in design and functionality (i.e., information presentation only) will be less expensive to develop. The more enhanced the functionality of the app, the more complicated the design, and the more expensive the app will be to build. This may mean that the end user must purchase the app, rather than download for free as is common with apps with less functionality and lower levels of interaction. Client expectations for a for-fee app may be higher than for a free app, especially with regard to unique functionality and "help" or service. Many users expect apps, particularly ones developed by land-grant university Cooperative Extension Services, to be free, which may influence their decision to purchase an app.

Native apps were initially written separately for each platform in their own programming language code, often costing $\$ 10,000$ or more per platform for basic "information presentation" apps. It is now possible to develop for Android and iOS platforms with less platform-specific code. Platform-neutral coding combined with building block app templates, which require minimal coding, have reduced the cost of app development. In a remarkably short time period, app development has mirrored website design and development, becoming a do-it-yourself process with little to no software programming expertise needed to develop the most basic apps. Just as websites revolutionized information availability and access to topical expertise, and webinars are now considered standard tools in the extension education toolbox, apps are emerging as an essential outreach tool, facilitating transfer of information and educational programming via a media tailored for on-the-go, "place-less" learning.

Several contractual issues should be considered when developing an app such as whether the app is free or for-fee, single- or multi-institution authorship, and if an internal or external programmer will be used. Developing a free app, particularly one that only includes authors from a single institution, will significantly reduce the contractual effort needed. Working with an in-house programmer, rather than contracting an external one, will also alleviate some need for a highly detailed contract that can otherwise be necessary to ensure the end-quality of the app rather than the programmer's profit margin remains the sole priority. Contractual responsibilities (contract preparation, authorization, etc.) can consume a substantial amount of predevelopment time and effort and must be factored into the overall time allotted for app development. 
When developing a for-fee app, the lead institution's research foundation or equivalent technology transfer office will likely become involved. More administrative time and coordination with the technology transfer office will be required for the first few apps developed at a given institution, especially if multiple institutions and codevelopers are involved as intra- and interinstitutional agreements must be agreed to, approved, and authorized by legal counsel and technology transfer entities at all institutions involved. Navigating administrative issues for a large multi-institution for-fee app can take 6 months or longer, while app development can take 4 months to 1 year (or more), depending on the scope of the app, number of subject matter experts, and programming complexity. For this reason, developing an app solely to address a current, urgent topic may not be a wise use of resources, as the significance of the topic may have diminished before the app is launched. Administrative time and expense of legal agreements, especially if the programming is outsourced, can also constitute major expenses.

If the programmer is external, a detailed contract for app development is necessary. The contract will ensure that the requirements and features are clearly communicated, stages of progress are clearly defined, acceptance of each stage is defined, both parties agree on how and what files will be submitted before final payment, and responsibility and procedures for troubleshooting are addressed. The contract should include the overall time frame in which both parties agree to complete the app and clearly state if ongoing maintenance for compatibility with new mobile device operating systems after the app is released as well as general trouble-shooting will be included in the contract. Clarifying how malfunctions and end-user issues are handled is essential, particularly for apps that are more than information presentation. In addition, the contract should address intellectual property protection for the app concept and any proprietary programming code. For example, can the software firm build a competing app with the same subject matter for another customer? Can the software firm build another app populated with different subject matter but with format and functionality identical to your app?
Hosting fees are one additional consideration for app development and should be discussed during preliminary conversations with potential programmers and addressed in the contract. Hosting and maintenance to ensure app integrity when new operating systems are released can increase overall costs. While universities have the capacity to host websites, web apps may require specialized software and dedicated servers. Native apps may also require devoted storage or computing capacity accessed via a web service, such as that required for complex calculations or user data entry/ archival function, that institutional information technology may not currently be able to accommodate. For example, IPMPro has hundreds of images that are housed in a server so that storage space on the mobile device is not consumed. IPMPro also allows growers to enter information for pesticide application records, which is archived on a server and accessed using a web service. Similar to a traditional website, content review, updates, and maintenance are recurring issues that should be addressed early in the process with all team members.

The TRUe COST OF APP DEVELOP. MENT. The cost of developing an app includes not only the software programming and administrative responsibilities, but also the time spent by the subject matter experts planning, designing, and testing the user interface, writing and editing text, as well as photographing, selecting, and editing images. In fact, depending on the complexity of the app and the uniqueness of the information contained within the app, this may represent a substantial cost in addition to the programming expense. For example, codeveloper JC Chong spent $\approx 270 \mathrm{~h}$ developing a degree-day based matrix to predict arthropod alert dates for numerous user locations for IPMPro. After the framework is developed, populating the app database can also represent a significant labor expense.

Time invested by the lead developer can be broken down into four main categories: 1) administrative (contract negotiation etc.), 2) managing content development (coordinating content preparation, editing, and upload), 3 ) public relations (preparing press releases, final testing, and managing the launch), and 4) troubleshooting and maintenance (Fig. 1). In

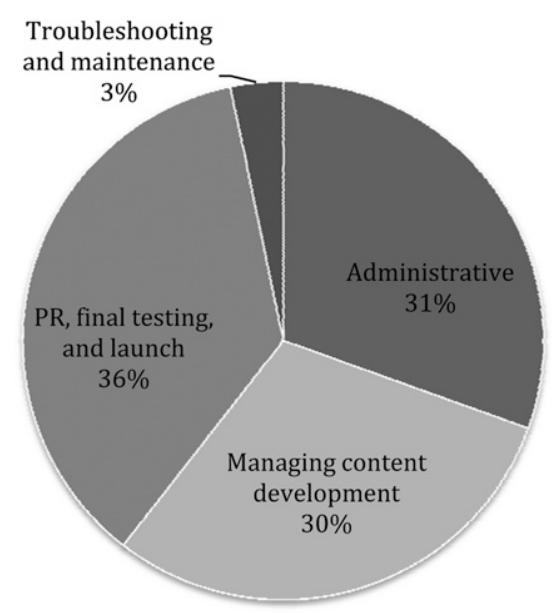

Fig. 1. Time commitment for lead developer during the development of a multi-institution, multifunctional app, IPMPro from idea conception through the month following launch (491 h total) $(\mathrm{PR}=$ public relations $)$.

addition, various stages of testing and evaluation occur continually from the earliest app development steps to postlaunch and, thus, reflect an ongoing time commitment. The greatest concentration of development hours for IPMPro was required just before launch due to promotional preparations, final testing, and the launch itself-a total of $170 \mathrm{~h}$.

App development responsibilities will likely take precedence over traditional extension programming, research, or publishing, so developing an app may not be an ideal pursuit at all career stages. The professional "return on investment" for developing an app may diminish as they become more commonplace in extension; however, institutional and peer expectations of app development may become greater. In addition, unlike many extension programs or research projects, which have a clear beginning and end, an app, particularly a for-fee app, is an ongoing professional and financial commitment. Although number of app downloads and revenue from app downloads are easily tabulated, current technology does not facilitate assessing changes in client behavior or the economic, environmental, and/or social impact of client use of information provided by the app, as would be desirable for documenting impact.

Developing in absentia. Can online or overseas app development firms be held accountable to the same degree as those based locally? 
A typical rate for app development in the United States may range from $\$ 100$ to $\$ 150$ per hour and higher, whereas the average overseas rate may be closer to $\$ 30$ per hour. Therefore, a substantial financial incentive exists to hire a software programmer from overseas or an online development firm. The inability to interact in person changes the nature of the business relationship. A local development company may provide the best of both worlds, offering a reasonable price point by outsourcing some programming tasks and working to protect its local reputation. Internships or other collaborations with computer science students, under the direct supervision of an experienced faculty member, are also options for developing a basic app.

Mobile device apps are a novel mechanism by which extension professionals can convey information to their clientele. Mobile device apps offer the flexibility of reaching people regardless of location and time of day and, unlike paper publications, can be updated at any time and can "push" information, allowing extension professionals the ability to quickly address emerging issues. App development is a multistep process that requires not only leadership, time and financial resources but also programming and subject matter experts. Planning and development may take six to 18 months or longer, but the reward can be a truly revolutionary approach to extension programming, making timely information available to our clients' with the touch of a fingertip.

\section{Literature cited}

Bradley, T. 2010. Managing smartphones in your business. 12 May 2013. <http://www.washingtonpost.com/ wp-dyn/content/article/2010/06/20/ AR2010062003850.html>.

Drill, S. 2012. Mobile application for extension. J. Ext. 50(5). 12 May 2013. <http://www.joe.org/joe/2012october/ pdf/JOE_v50_5ttl.pdf $>$.

Fulcher, A., J-H. (JC) Chong, S. White, J. Williams-Woodward, C. Adkins, K. Braman, M. Chappell, J. Derr, W. Dunwell, S. Frank, S. Gill, F. Hale, W. Klingeman, A. LeBude, J. Neal, K. Rane, and A. Windham. 2012. IPMPro app. Android, iPhone, iPod Touch, iPad. 12 May 2013. <https:// itunes.apple.com/us/app/ipmpro/ id526045862? $\mathrm{mt}=8>$ and $<$ https://play. google.com/store/search?q=IPMPro $>$.

Fulcher, A., S. White, J-H. (JC) Chong, J. Neal, J. Williams-Woodward, C. Adkins, K. Braman, M. Chappell, J. Derr, W Dunwell, S. Frank, S. Gill, F. Hale, W. Klingeman, A. LeBude, K. Rane, and A Windham. 2013. Testing, promoting, and launching a mobile application as an extension education tool: A case study using IPMPro. HortTechnology 23:407-410.

Guenthner, J.F., and B.G. Swan. 2011. Extension learners' use of electronic technology. J. Ext. 49(1). 12 May 2013. <http://www.joe.org/joe/201lfebruary/ pdf/JOE_v49_la2.pdf>.

Intaravanne, Y. and S. Sumriddetchkajorn. 2012. BaiKhao (rice leaf) app: A mobile device-based application in analyzing the color level of the rice leaf for nitrogen estimation. Proc. Optoelectronic Imaging Multimedia Technol. II. 85580F. 12 May
2013. <http://dx.doi.org/10.1117/ $12.2001120>$.

LaBelle, C. 2011. Place-based learning and mobile technology. J. Ext. 49(6). 12 May 2013. <http://www.joe.org/joe/ 2011 december/pdf/JOE_v49_6iwl.pdfs.

Luo, L. 2010. Native or web application: How to best deliver content and services to your audiences over the mobile phone. Global Intelligence Alliance, Helsinki, Finland.

McCullough, P.E., F.C. Waltz, Jr., W. Hudson, and A.D. Martinez-Espinoza. 2011. Turfgrass management at your fingertips: Information delivered through "smart" phone technology. J. Ext. 49(3). 12 May 2013. <http://www.joe.org/ joe $/ 201$ ljune/tt10.php>.

Moscaritolo, A. 2013. Apple iOS tops U.S. smartphone market. 12 May 2013. <http://www.pcmag.com/article2/ $0,2817,2413934,00 . \mathrm{asp}>$.

Neilsen Co. 2012a. Smartphones account for half of all mobile phones, dominate new phone purchases in the U.S. 12 May 2013. <http://www.nielsen.com/us/en/ newswire $/ 2012 /$ smartphones-accountfor-half-of-all-mobile-phones-dominatenew-phone-purchases-in-the-us.html>.

Neilsen Co. 2012b. Two thirds of new mobile buyers now opting for smartphones. 12 May 2013. <http://blog.nielsen.com/ nielsenwire/online_mobile/two-thirdsof-new-mobile-buyers-now-opting-forsmartphones $/>$

Walter, J., J. Davey, and J. Caldwell. 2011. Smartphones a big trend. Successful Farming 109:32-36. 